\title{
Rate-dependent ductile fracture under plane strain tension: experiments and simulations
}

\author{
Vincent Grolleau ${ }^{1,3, *}$, Vincent Lafilé ${ }^{1}$, Christian C. Roth ${ }^{3}$, Bertrand Galpin ${ }^{1,2}$, Laurent Mahéo ${ }^{1,2}$, and Dirk Mohr ${ }^{3,4}$ \\ ${ }^{1}$ Univ. Bretagne Sud, FRE CNRS 3744, IRDL, F-56100 Lorient, France \\ ${ }^{2}$ LMM, Ecole Militaire de Sinat Cyr Coëtquidan, F-56381 Guer, France \\ ${ }^{3}$ Department of Mechanical and Process Engineering, ETH Zurich, Zurich, Switzerland \\ ${ }^{4}$ Impact and Crashworthiness Laboratory, Department of Mechanical Engineering, Massachusetts Institute of Technology, Cambridge, \\ MA, USA
}

\begin{abstract}
Among all other stress states achievable under plane stress conditions, the lowest ductility is consistently observed for plane strain tension. For static loading conditions, V-bending of small sheet coupons is the most reliable way of characterising the strain to fracture for plane strain tension. Different from conventional notched tension specimens, necking is suppressed during V-bending which results in a remarkably constant stress state all the way until fracture initiation. The present DYMAT talk is concerned with the extension of the V-bending technique from low to high strain rate experiments. A new technique is designed with the help of finite element simulations. It makes use of modified Nakazima specimens that are subjected to V-bending. Irrespective of the loading velocity, plane strain tension conditions are maintained throughout the entire loading history up to fracture initiation. Experiments are performed on specimens extracted from aluminum 2024-T3 and dual phase DP450 steel sheets. The experimental program includes quasi static loading conditions which are achieved on a universal testing machine. In addition, high strain rate experiments are performed using a specially-designed drop tower system. In all experiments, images are acquired with two cameras to determine the surface strain history through stereo Digital Image Correlation (DIC). The experimental observations are discussed in detail and also compared with the numerical simulations to validate the proposed experimental technique
\end{abstract}

\section{Introduction}

Among all other stress states achievable under plane stress conditions, the lowest ductility of metallic materials is consistently observed for plane strain tension. This motivates an ongoing quest for reliable experimental techniques to determine the strain to fracture of sheet metals under Plane Strain Tension loading conditions, from quasistatic to high strain rates. Unfortunately, conventional PST geometries are prone to fracture initiation from the free edges of the specimen and non-proportional loading histories (i.e. varying triaxiality and Lode angle parameters) during plastic deformation [1-3], see for example the NT2 notched geometry on Fig.1. This led to the use of the VBending test by Roth and Mohr [4], Fig.1. Different from conventional notched tension specimens, necking is suppressed during V-bending which results in a remarkably constant stress state all the way until fracture initiation. This test has two main drawbacks. First, the maximum strain is limited from geometrical consideration. Assuming that the neutral bending axis coincides with the sheet mid-plane, the equivalent plastic strain in a bent sheet of thickness $\mathrm{t}$ and inner radius of curvature $\mathrm{R}$ is

$$
\bar{\varepsilon}=\frac{1}{\sqrt{3}} \ln \left(\frac{R+t}{R+t / 2}\right)
$$

Second, the increase of the relative speed between the blade and the specimen is necessary in order to perform high strain rate experiments, but this would lead to bending spurious oscillations of the specimen. To overcome these difficulties, a new PST test is proposed. It makes use of modified Nakazima specimens that are subjected to V-bending, i.e. stretch-bending.
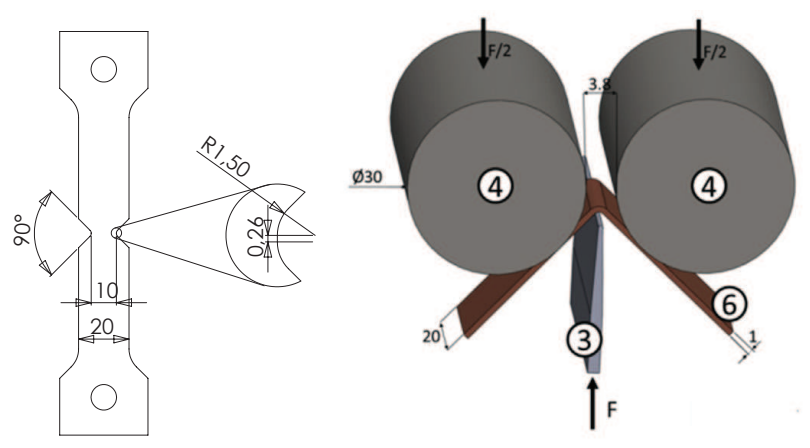

Fig. 1. Left, design of the NT2 tensile notched specimen used for PST test on DP450 steel, derived from [2].

Right, basic design principle of the V-Bending test, from [4] with (3) the knife-like punch, (4) the rollers and (6) the $60 \mathrm{~mm}$ long and $20 \mathrm{~mm}$ wide specimen.

\footnotetext{
*e-mail: vincent.grolleau@univ-ubs.fr
} 


\section{Dual Hole Plane Strain Tension test apparatus}

In the proposed plane strain test presented Fig.2, the sheet material is subjected to stretch bending. In close analogy with the bending test [4], the active part of the punch is a $r_{p}=1 \mathrm{~mm}$ radius and $l_{p}=12 \mathrm{~mm}$ long cylinder machined along the edge of a dihedral end of a $d_{p}=12 \mathrm{~mm}$ diameter hard steel bar. This cylinder is in contact with the sample along the ligament. The sample is a $60 \mathrm{~mm}$ diameter disk, 8 holes are machined on the periphery for clamping purpose. The inner diameter of the clamping die is diameter $D$. Two symmetric and chamfered holes, diameter $d$, are machined along a diameter of the specimen, the edge to edge distance is $l$, the length of the ligament, where contact between punch and sample takes place on the non chamfered sample face.

In the quasi static version of the test, the presented device Fig.2 is inserted in a universal testing machine, keeping the punch stationary, while moving the base matrix. This configuration has the advantage that the surface strain field can be measured by means of stereo DIC without any spatial resolution limitations imposed by the limited depth of focus of the optical system. For quasi-static experiments, images are taken with two 5MP cameras in conjunction with a 1:1 macro lens (f $2.8,100 \mathrm{~mm}$ ) at a frequency of $1 \mathrm{~Hz}$, and resulting to a spatial resolution of approximately $10 \mu \mathrm{m} /$ pixel.

In the case of high strain rate experiments, a drop weight tower is used. The sample is clamped in the center of the $9 \mathrm{~kg}$ free flying projectile. A quarter view of the system is presented Fig.3. The punch is stationary, the active part is not modified from the quasi static experiments, but the body of the punch is adapted to the specially designed load cell, so-called sensing block (ref 5 Fig.3), a high bandwidth strain gage load cell [5]. Images are taken with two $1 \mathrm{MP}$ high speed cameras in conjunction with a 1:1 macro lens (f $2.8,105 \mathrm{~mm}$ ) at a frequency of $50 \mathrm{kHz}$, resulting to an image size of $512 \times 272$ pixels and a spatial resolution of approximately $85 \mu \mathrm{m} /$ pixel.

\section{Material parameters identification and numerical simulation}

Two different materials, a $1.2 \mathrm{~mm}$ thick aluminium alloy AA2024-T351 and a $0.85 \mathrm{~mm}$ thick dual phase steel DP450 are examined in the present study.

Quasi static tests are performed on a $100 \mathrm{kN}$ universal testing machine equipped with custom made high pressure clamps under displacement control, a strain rate of the order of $0.001 / s$ and planar digital image correlation (VIC 2D). A first set of quasi-static experiments is performed to identify the basic plasticity response of the materials. They comprise Uniaxial Tension (UT) along the rolling $\left(0^{\circ}\right)$, diagonal $\left(45^{\circ}\right)$ and transverse $\left(90^{\circ}\right)$ direction and $200 \mathrm{~mm}$ and $100 \mathrm{~mm}$ diameter equi-biaxial bulge tests.

A second set of experiments is carried out to calibrate the fracture model and validate the plasticity model. It comprises notched tension NT20 and NT6 specimens with

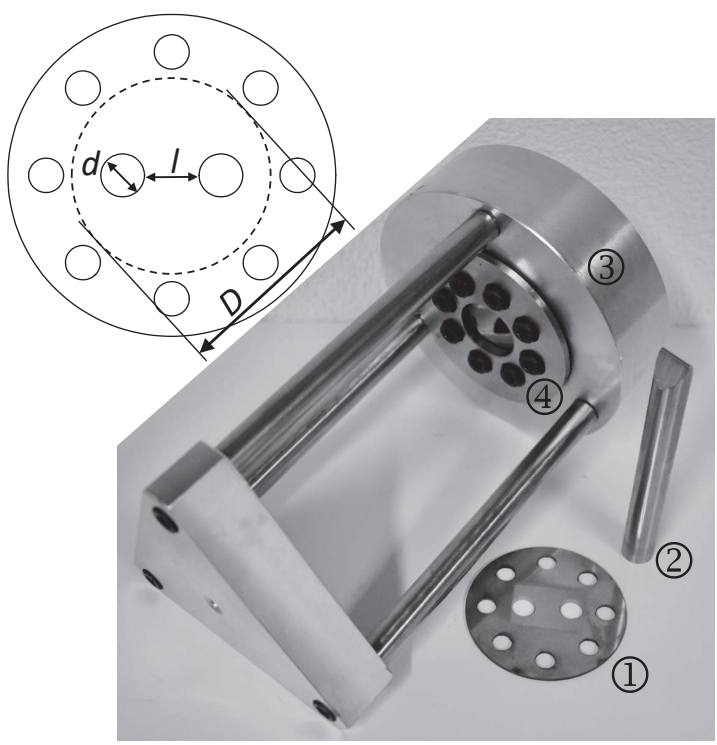

Fig. 2. Design of the DHPST sample and photo of the DHPST device, with (1) an undeformed $60 \mathrm{~mm}$ diameter specimen, (2) the $1 \mathrm{~mm}$ edge radius punch, (3) the base matrix and (4) the clamping die. The clamped specimen is presented in the fractured configuration.

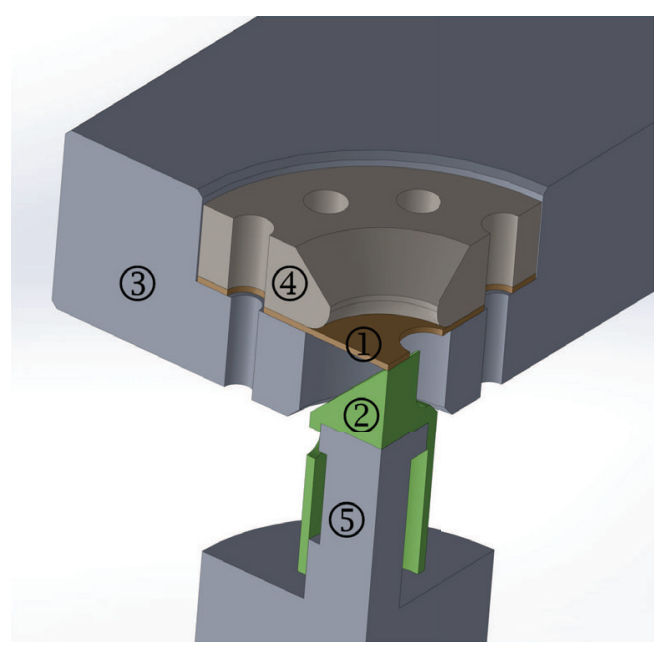

Fig. 3. Quarter view of the high strain rate DHPST device, with (1) the $60 \mathrm{~mm}$ diameter specimen, (2) the $1 \mathrm{~mm}$ edge radius cap, (3) the $9 \mathrm{~kg}$ free flying base matrix, (4) the clamping die and (5) the specially designed sensing-block load cell.

a $20 \mathrm{~mm}$ wide shoulder section and notch cutouts with a radius of $R=20 \mathrm{~mm}$ ( $R=6.67 \mathrm{~mm}$ resp.), reducing the width of the gage section to $10 \mathrm{~mm}$ at the specimen center. It comprises also central hole $\mathrm{CH}$ tension specimens, with $20 \mathrm{~mm}$ wide shoulder section and a $6 \mathrm{~mm}$ circular cutout in the center.

A third set of tests is carried out to calibrate the fracture model. It comprises a $12.7 \mathrm{~mm}$ equi-biaxial mini punch test PU for the identification of the fracture strain at a theoretical constant stress triaxiality value of $\eta_{P U}=2 / 3$; shear experiments $\eta_{S H}=0.0$ and plane strain tension test $\eta_{P S T}=1 / \sqrt{3}$. In the case of AA2024 aluminium alloy, the V-Bending test is used, while NT2 specimen exhibited 
initiation of fracture from the edge. On the contrary, PST tests are successfully performed using the NT2 specimen in the case of DP450 steel, for which the fracture strain is higher than the maximum strain achievable using the V-Bending test for this material thickness of $0.85 \mathrm{~mm}$.

The last set of experiments is dedicated to the identification of the strain rate sensitivity of the two materials. Intermediate strain rate NT20 and NT6 experiments are conducted using the previous universal testing machine at a strain rate of the order of $1 / s$. High strain rate NT20 and NT6 tests are performed on a split Hopkinson pressure bar (SHPB) system equipped with a load inversion device for tensile testing, $6 \mathrm{~m}$ long and $20 \mathrm{~mm}$ diameter steel bars and stricker, at strain rate about $100 / s$, see [6, 7].

To accurately capture the different material behaviours, two different constitutive modelling approaches are chosen. For the Dual Phase steel, a non associated plasticity model is used, combining a von Mises yield surface with isotropic hardening, and a non associated Hill' 48 flow rule, see [7, 8]. For the AA2024 aluminium alloy, an extension of the anisotropic associated plasticity Barlat'YLD2000-2D model [9] is used, see [10, 11]. For both materials, an isotropic hardening law is used to describe the relationship between the deformation resistance and the equivalent plastic strain $\bar{\varepsilon}_{p l}$ combining Swift and Voce laws. Strain rate sensitivity was found to be negligible for the aluminium alloy, while a Johnson-Cook sensitivity coefficient is taken into account in the case of DP450 steel. The force-displacement curves for two D450 different specimens are shown all the way to fracture Fig.4, along with the evolution of the local strain. Displacement and surface strain are based on $30 \mathrm{~mm}$ long and $1 \mathrm{~mm}$ long respectively DIC virtual extensometers.

Finite element simulations are performed of each experiment, using user material subroutines. Exploiting the symmetry of the specimen, only one eighth of the specimen is modelled using a mesh size of the order of $60 \mu \mathrm{m}$. The accuracy of the identified behaviour can be seen Fig. 4 .
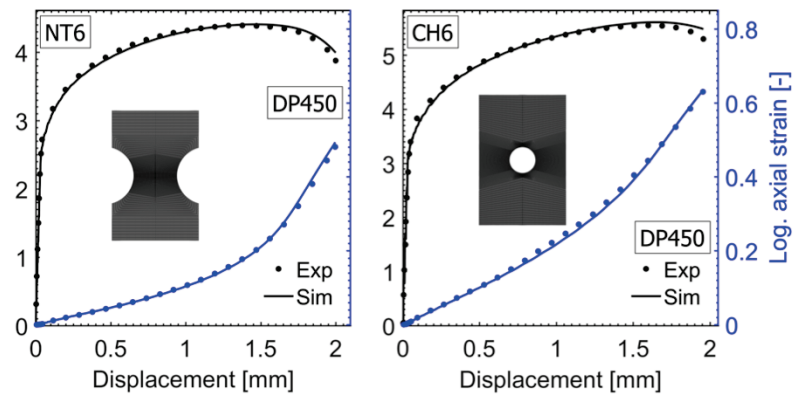

Fig. 4. Experimental (dots) and simulation (solid line) results for DP450 tensile specimen NT6 and CH6. Force-displacement curves are in black, central surface logarithmic axial strain versus displacement curves are in blue.

The Hosford-Coulomb fracture initiation model [8] is used to describe the onset of fracture. It defines the equivalent plastic strain to failure for proportional loading $\bar{\varepsilon}_{f}^{p r}[\eta, \bar{\theta}]$ as a function of the stress triaxiality $\eta=\sigma_{m} / \bar{\sigma}$ and the Lode angle parameter $\bar{\theta}=1-\frac{2}{\pi} \arccos \left[\frac{27 J_{3}}{2 \bar{\sigma}^{3}}\right] \epsilon$ $[-1 ; 1]$. This damage indicator predicts the onset of fracture once the condition

$$
\int_{0}^{\bar{\varepsilon}_{f}} \frac{d \bar{\varepsilon}_{p l}}{\bar{\varepsilon}_{f}^{p r}[\eta, \bar{\theta}]}=1
$$

is met, where equivalent plastic strain to failure for proportional loading $\bar{\varepsilon}_{f}^{p r}[\eta, \bar{\theta}]$ reads as

$$
\bar{\varepsilon}_{f}^{p r}[\eta, \bar{\theta}]=b(1+c)^{1 / n}\left[\{F(a, \bar{\theta})\}+c\left(2 \eta+f_{1}+f_{3}\right)\right]^{1 / n}
$$

with

$$
F(a, \bar{\theta})=\frac{1}{2}\left\{\left(f_{1}-f_{2}\right)^{a}+\left(f_{2}-f_{3}\right)^{a}+\left(f_{3}-f_{1}\right)^{a}\right\}^{1 / a}
$$

and

$$
\begin{gathered}
f_{1}=\frac{2}{3} \cos \left[\frac{\pi}{6}(1-\bar{\theta})\right] \quad, \quad f_{2}=\frac{2}{3} \cos \left[\frac{\pi}{6}(3+\bar{\theta})\right] \\
\text { and } f_{3}=-\frac{2}{3} \cos \left[\frac{\pi}{6}(1+\bar{\theta})\right]
\end{gathered}
$$

Three parameters control the fracture locus, namely the Hosford exponent $a$ controlling the influence of the Lode angle parameter, the uni-axial and bi-axial strain to fracture $b$ and the friction coefficient $c$ controlling the effect of stress triaxiality on the strain to fracture, while $n=0.1$ is a fixed transformation coefficient. A view of the identified fracture locus as a function of triaxiality is presented Fig.5 for each material.

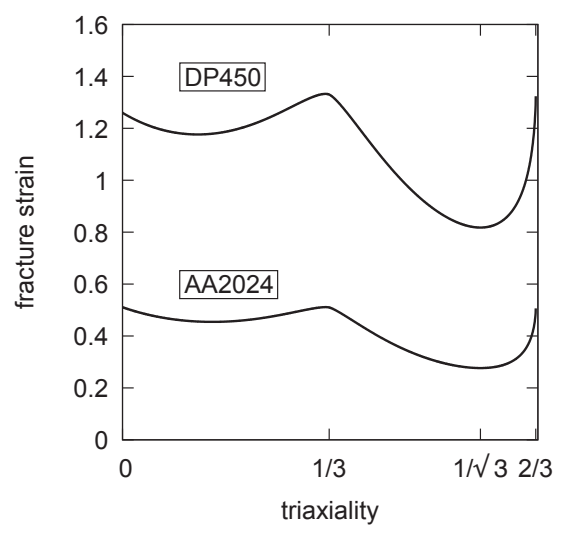

Fig. 5. Illustration of the fracture locus for plane stress proportional loading of the DP450 and AA2024 materials as a function of the stress triaxiality.

\section{Design of the Dual Hole Plain Strain Tension (DHPST) test}

Using the identified materials parameters, a numerical study of four geometrical parameters of the DHPST test is conducted, the punch radius $r_{p}$, the ligament length $l$, the hole diameter $d$, the chamfer size $c_{z}$. The clamping diameter is considered as a fixed value $D=30 \mathrm{~mm}$. The influence 
of the punch sample friction $f_{\text {ric }}$ and sample thickness $t$ is also studied. A quarter of the sample is simulated with Ls-dyna. The objective of the optimisation process is to obtain:

- a proportional loading up to fracture under plane strain tension condition, defined from both the strain state, with a major to minor surface strain ratio higher than 5 [1], as well as the stress state, with a triaxiality as close as possible to the theoretical value $\eta_{P S T}=1 / \sqrt{3}$

- a constant value of the plane strain tension condition along the ligament in order to have a straightforward measurement of the strain to fracture from DIC

- a valid geometry for any combination of fracture strain in the range $[0.3 ; 1$.$] and material thickness in the range$ $[0.5 ; 2] \mathrm{mm}$.

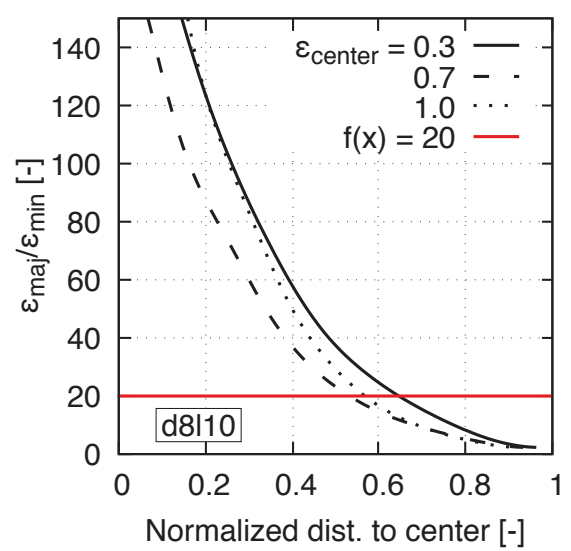

Fig. 6. Evolution of the major to minor log strain ratio along the ligament.

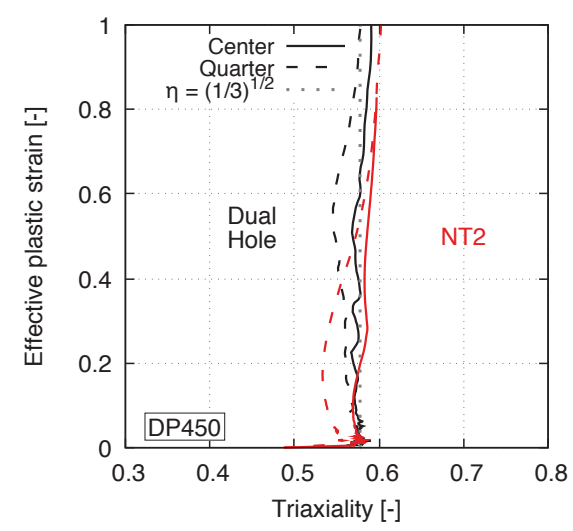

Fig. 7. Effective strain versus triaxiality at two locations along the ligament in the case of NT2 (red) and DHPST (black) geometries.

The optimisation of the DHPST geometry leads to the following geometrical parameters $l=10 \mathrm{~mm}, d=8 \mathrm{~mm}$, $r_{p}=1 \mathrm{~mm}$ and a chamfer depth equal to half the sample thickness. The numerical study shows that the samplepunch friction, modelled using Coulomb's friction, has a negligible influence. The following pictures are obtained using these optimised parameters.

Fig. 6 shows the evolution of the ratio of the logarithmic major to minor strains along the ligament in the case of DP450 DHPST test for three different effective strains in the center of the specimen. The ratio is higher than 20 along more than $60 \%$ of the ligament length, and higher than 5 along $80 \%$ of the ligament, whatever the strain level in the center.

Fig. 7 shows the evolution of the effective strain as a function of the triaxiality at the center and at mid distance between the center and the edge, i.e. for a normalized distance to center value of 0.5 . The plot shows that the loading path remains close to the theoretical plane strain tension path.

The evolution of the effective strain along the ligament is plotted for three different time points, each referring to a distinct strain level at the specimen center Fig.8. Even though the strain is not perfectly constant along the ligament, it varies only by 0.05 over the inner half of the ligament, a variation in the order of the experimental noise.

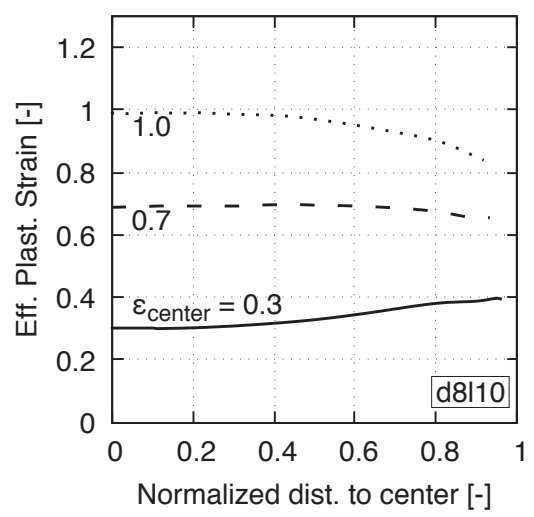

Fig. 8. Evolution of the effective strain along the ligament.

\section{Experimental results using DHPST}

The two following plots, Fig.9 and 10 show results from quasi static DHPST experiments. Herein the experimental and numerical effective log strain, and the numerical triaxiality are plotted along the normalised position on the ligament's outer surface at the time of fracture of the specimen. The graphs are presented along with the effective strain contour plots from DIC.

From the practical usage point of view, one can see that the effective strain is almost constant along the central half of the ligament, and thus the experimental evaluation of the effective strain to fracture is straightforward. This observation is even more pronounced in the case of the DP450. Its high fracture strain level of more than 0.7 leads to a remarkable uniformity of the strain along the ligament as already shown Fig.8. A modification of the DHPST geometry, changing the ligament length and the hole diameter, can be performed to obtain a similar level of strain uniformity for lower ductilities. The importance 

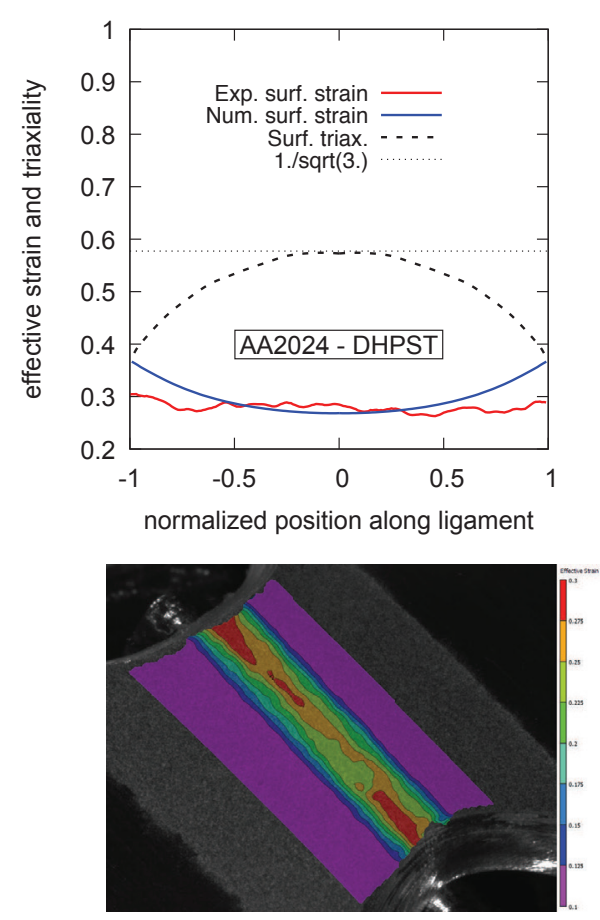

Fig. 9. DHPST test of the AA2024 alloy. Effective strain and triaxiality evolution along the ligament at onset of fracture. Effective log strain contours from DIC, maximum fringe value is 0.3 , fringe step is 0.025 in strain.
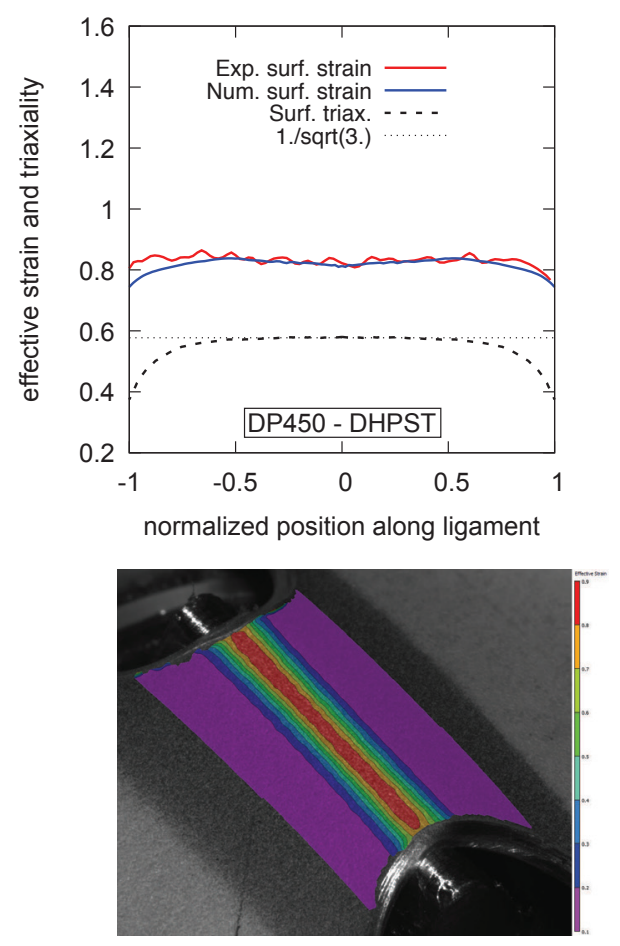

Fig. 10. DHPST test of the DP450 steel. Effective strain and triaxiality evolution along the ligament at onset of fracture. Effective $\log$ strain contours from DIC, maximum fringe value is 0.9 , fringe step is 0.1 of the uniform strain field can be appreciated when comparing to the evolution of the mechanical fields along the ligament in the case of NT2 geometry, Fig.11, exposing an amplitude of about 0.3 in strain along the ligament.



Fig. 11. NT2 test of the DP450 steel. Effective strain and triaxiality evolution along the ligament at onset of fracture, at mid plane thickness (adshed line) and outward surface (solid line). The location of first cracks along the ligament are highlighted in grey.

During high strain rate DHPST tests, performed within a drop weight tower, the limited spatial resolution of the DIC preclude a direct measurement of the effective strain all the way to fracture. Thus, an hybrid numerical and experimental technique is necessary to analyse the test. This method relies on the measurement of the load during the test obtained from the specially designed load cell. Fig.12 shows the evolution of the measured load for two DP450 specimens as a function of time, with a very good reproducibility of the measure. It also relies on the DIC fields and on the measurements of the position, the speed and the acceleration of the free flying base matrix of the DHPST apparatus.

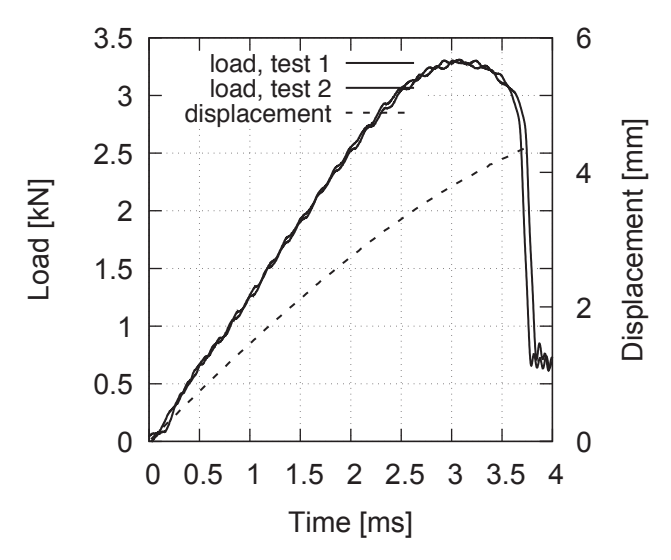

Fig. 12. Evolution of the load versus time for two tests, and displacement versus time during High Strain Rate DHPST tests on DP450. 


\section{Conclusion}

Among all other stress states achievable under plane stress conditions, the lowest ductility is consistently observed for plane strain tension. For static loading conditions, Vbending of small sheet coupons is the most reliable way of characterising the strain to fracture for plane strain tension, but its extension to high strain rate is limited by spurious oscillations of the sample. The aim of the present work is to design an extension of the V-bending technique from low to high strain rate experiments.

A new test, a so-called Dual Hole Plane Strain Tension DHPST has been designed by means of finite element simulations. It makes use of modified Nakazima specimens subjected to V-bending. Irrespective of the loading velocity, plane strain tension conditions are maintained throughout the entire loading history up to fracture initiation.

Experiments have been performed on specimens extracted from aluminum 2024-T3 and dual phase DP450 steel sheets. The experimental program includes quasi static loading conditions which have been achieved on a universal testing machine. In addition, high strain rate experiments have been performed using a drop tower system and a specially-designed load cell. The main advantages of the proposed geometry are the constant stress state all the way to fracture and the uniformity of the mechanical fields along the ligament, leading to a straightforward determination of the effective strain to fracture for quasi-static loading, and from hybrid analysis at high strain rate.

Vincent Lafile was the recipient of a Doctoral fellowship from French government, Ministère de la Défense, Direc- tion Générale de l'Armement. We thank Mr Penin from Mat\&Sim company for his technical support. These supports are gratefully acknowledged.

\section{References}

1. J. Dournaux, S. Bouvier, A. Aouafi, P. Vacher, Materials Science and Engineering: A 500, 47 (2009)

2. P. Flores, V. Tuninetti, G. Gilles, P. Gonry, L. Duchêne, A.M. Habraken, Journal of Materials Processing Technology 210, 1772 (2010)

3. D. Banabic, in Sheet Metal Forming Processes (Springer, 2010), pp. 14-204

4. C.C. Roth, D. Mohr, International Journal of Plasticity 79, 328 (2016)

5. B. Galpin, V. Grolleau, S. Umiastowski, G. Rio, L. Mahéo, International Journal of Crashworthiness 13, 139 (2008)

6. M. Dunand, G. Gary, D. Mohr, Experimental Mechanics 53, 1177 (2013)

7. C.C. Roth, D. Mohr, International Journal of Plasticity 56, 19 (2014)

8. D. Mohr, S.J. Marcadet, International Journal of Solids and Structures 67-68, 40 (2015)

9. F. Barlat, J. Brem, J. Yoon, K. Chung, R. Dick, D. Lege, F. Pourboghrat, S.H. Choi, E. Chu, International Journal of Plasticity 19, 1297 (2003)

10. M. Dunand, A.P. Maertens, M. Luo, D. Mohr, International Journal of Plasticity 36, 34 (2012)

11. M.B. Gorji, D. Mohr, International Journal of Mechanical Sciences 137, 105 (2018) 